\title{
COMMENT
}

\section{Commentary on "Oxygen desaturations in the early neonatal period predict development of bronchopulmonary dysplasia" by Fairchild et al.}

\author{
Natalie L. Davis ${ }^{1}$ \\ Pediatric Research (2019) 85:927-928; https://doi.org/10.1038/s41390-019-0358-z
}

Supplemental oxygen is simultaneously the best friend and worst enemy of very low birth weight (VLBW) preterm infants. Without the ability to provide supplemental oxygen, many would not survive. Studies have shown increased incidence of dangerous comorbidities such as severe necrotizing enterocolitis in those infants with lower oxygen saturation (SpO2) targets ${ }^{1,2}$. However, oxygen exposure also leads to significant comorbidities such as retinopathy of prematurity $(\mathrm{ROP})^{1,2}$ and bronchopulmonary dysplasia (BPD). Determining when to increase the fraction of inspired oxygen (FiO2), or more to the point determining which desaturation events matter, remains challenging. The goal is to prevent and treat desaturation events that could lead to detrimental outcomes all while avoiding hyperoxia in this vulnerable population. In the study entitled "Oxygen desaturations in the early neonatal period predict development of bronchopulmonary dysplasia" by Fairchild et al. ${ }^{3}$ in this issue of Pediatric Research, the authors sought to both describe the natural course of desaturation and bradycardic events in VLBW infants in the first month, as well as to determine if these events are clinically significant.

The authors' first aim was to describe the natural course of bradycardia and desaturations in a large cohort of 500 VLBW infants over the first 28 days of life. They did this independently of apnea as many of these events occur in the absence of central apnea and may also be related to obstruction, chronic lung disease, and intra-cardiac shunts. On average, infants spent $>45$ min daily in either a bradycardia or desaturation event, or both. Interestingly, VLBW infants spent on an average only $3.3 \%$ of their day in a desaturation event (defined in this study as $<80 \%$ for $\geq 10 \mathrm{~s}$ ). This varied widely and was significantly higher in lower birth weight infants, with some of the extremely low birth weight (ELBW) infants spending $>10 \%$ of their day desaturated. The median duration of desaturation events was $30 \mathrm{~s}$, indicating that many were short in duration. The frequency increased over time and peaked around 3 weeks of life. There were no data on which of these events were acted upon by the clinical team ${ }^{3}$.

But why does this matter? Besides alarm fatigue for the staff, what does this mean for the health and safety of our infants? It has been previously shown that intermittent hypoxic $(\mathrm{IH})$ events are common in preterm infants in the first 2 months of life, peaking at 2-4 weeks and declining towards $6-8$ weeks $^{4}$. Infants often had upwards of $50-100 \mathrm{IH}$ events per day ${ }^{4,5}$. Determining whether these events (and which of these events) are clinically significant and should be acted upon is of the utmost importance since our interventions are far from benign. Increasing $\mathrm{FiO} 2$ increases exposure to oxygen free radicals and risk of inflammation and injury to the developing lung and retina. Opening the isolette to stimulate a baby increases sound and temperature stressors. Because of this, the authors' second aim was to assess this very question, whether oxygen desaturation events within the first 28 days (with or without bradycardia) can be used to predict later comorbidities and length of stay.

While accounting for known risk factors such as gestational age, birth weight, and respiratory support requirements, the authors found that accurate early data on desaturation events (frequency and duration) enhanced the ability to identify those VLBW infants at highest risk of developing BPD at postmenstrual age 36 weeks, as well as ROP, severe intraventricular hemorrhages (IVH), and prolonged NICU length of stay. It is important to note that the definition of a "desaturation event" in this study $(<80 \%$ for $\geq 10 \mathrm{~s})$ is conservative and does not mean that milder events have no clinical impact on outcomes. In addition, outcomes such as BPD, ROP, and IVH have multifactorial etiologies besides desaturation events. Risk factors such as hypotension, hemodynamically significant patent ductus arteriosus, sepsis events, and time spent with hyperoxia also contribute to these comorbidities and are important to take into account as well when assessing risk. Finally, a limitation of any study evaluating episodes of hypoxia and the association with BPD is the difficulty differentiating whether $\mathrm{IH}$ events have an effect on BPD incidence or are simply a marker of the underlying disease process.

But it does appear that obtaining accurate data on $\mathrm{SpO} 2$ trends could change clinical care by assisting in treatment decisions aimed at decreasing incidence of desaturation events. Although this clearly needs more study, it is an intriguing finding. This begs the question-how accurate is our bedside data on $\mathrm{IH}$ events and can we use it to affect clinical care? In this study, the authors performed continuous pulse oximetry with an 8-s averaging time, obtaining multiple $\mathrm{SpO} 2$ data points per minute. In clinical practice, most NICUs rely on bedside nurse documentation or automatic download into the electronic medical record which often occurs no more frequently than every 10-15 min and relies on the specific $\mathrm{SpO} 2$ at the moment of documentation. Bedside reporting of apnea, bradycardia, and desaturation events in the medical record has been shown to be inaccurate, often markedly underestimating the frequency and duration of these events in neonates ${ }^{6-8}$. Compared to continuous pulse oximetry, bedside transcribed $\mathrm{SpO} 2$ data tend to over-document time spent within

${ }^{1}$ Division of Neonatology, Department of Pediatrics, University of Maryland School of Medicine, 110 S. Paca Street, 8th Floor, Baltimore, MD 21201, USA

Correspondence: Natalie L. Davis (Natalie.davis@som.umaryland.edu)

Received: 6 November 2018 Revised: 4 February 2019 Accepted: 26 February 2019

Published online: 5 March 2019 
the target range, and under-document both time spent hypoxic and hyperoxic ${ }^{9}$.

Although they found a significant discrepancy, many authors note that it is unclear what clinical significance these underrepresented, or "missed," events had on the patients and if their care would have changed if the data had been more accurate. However, frequency of $\mathrm{IH}$ events during the first 2 months of life has been shown to be significantly associated risk of laser ROP ${ }^{4}$. This study by Fairchild et al. ${ }^{3}$ also seems to indicate that oxygen desaturations in the first month of life are important, and that more precise, accurate data could be useful in risk stratification, early prediction of BPD and other serious comorbidities, and may eventually be useful in early treatment decisions.

As the authors point out, this level of precision in identifying hypoxia is not feasible in the majority of NICUs yet. However, more accurate quantitation of time spent within certain saturation parameters even early in the NICU course is attainable. Some pulse oximeters can be programed to display a SpO2 "histogram," which graphically represents the percentage of time spent in pre-set SpO2 ranges (such as $<85 \%, 86-90 \%, 91-95 \%, 96-100 \%$ ) over the prior $24 \mathrm{~h}$. Although this does not quantify IH events, it is a simple and non-invasive method of obtaining more accurate data on desaturation time than bedside nursing documentation. Assessing histogram parameters has been shown to predict successful weaning of respiratory support in VLBWs requiring positive pressure $^{10}$. Daily assessment of a $\mathrm{SpO} 2$ histogram is a simple way to identify not just number of "events" but time spent below and within critical thresholds for each infant. We cannot change gestational age or birth weight once an infant is born, but we can mitigate risk by using more objective assessments such as a daily $\mathrm{SpO} 2$ histogram to improve our awareness and ability to maintain infants within target ranges.

Up and coming modalities such as closed-loop automated $\mathrm{FiO}_{2}$ (A-FiO2) systems could, in theory, markedly improve time spent in target $\mathrm{SpO} 2$ ranges and decrease burden on bedside caregivers. Although the studies of A-FiO2 systems in preterm infants are heterogeneous for sample size, type of A-FiO2 system used, and duration of observation, they do demonstrate significant improvement in time spent within target ranges as well as decreased time spent above target ranges ${ }^{11}$. However, evidence for prevention of hypoxia episodes is less consistent, and none evaluate longer term outcomes in preterm infants ${ }^{11}$. Therefore, it is of the utmost importance that moving forward, research continues to focus on the clinical significance and optimal modalities to identify, prevent, and safely treat desaturation events in VLBW infants.

\section{ADDITIONAL INFORMATION}

Competing interests: The author declares no competing interests.

Publisher's note: Springer Nature remains neutral with regard to jurisdictional claims in published maps and institutional affiliations.

\section{REFERENCES}

1. Askie, L. M. et al. Effects of targeting lower versus higher arterial oxygen saturations on death or disability in preterm infants. Cochrane Database Syst. Rev. 4, CD011190 (2017).

2. Askie, L. M. et al. Association between oxygen saturation targeting and death or disability in extremely preterm infants in the neonatal oxygenation prospective meta-analysis collaboration. JAMA 319, 2190-2201 (2018).

3. Fairchild, D., Nagraj, V. P., Sullivan B. A., Moorman, J. R. \& Lake, D. E. Oxygen desaturations in the early neonatal period predict development of bronchopulmonary dysplasia. Pediatr. Res. (2018) https://doi.org/10.1038/s41390-0180223-5 [Epub ahead of print].

4. Di Fiore, J. M. et al. A higher incidence of intermittent hypoxemic episodes is associated with severe retinopathy of prematurity. J. Pediatr. 157, 69-73 (2010).

5. Martin, R. J., Wang, K., Koroglu, O., Di Fiore, J. \& Kc, P. Intermittent hypoxic episodes in preterm infants: do they matter? Neonatology 100, 303-310 (2011).

6. Vergales, B. D. et al. Accurate automated apnea analysis in preterm infants. Am. J. Perinatol. 31, 157-162 (2014).

7. Brockmann, P. E. et al. Under-recognition of alarms in a neonatal intensive care unit. Arch. Dis. Child Fetal Neonatal Ed. 98, F524-F527 (2013).

8. Amin, S. B. \& Burnell, E. Monitoring apnea of prematurity: validity of nursing documentation and bedside cardiorespiratory monitor. Am. J. Perinatol. 30, 643-648 (2013).

9. Ruiz, T. L., Trzaski, J. M., Sink, D. W. \& Hagadorn, J. I. Transcribed oxygen saturation vs oximeter recordings in very low birth weight infants. J. Perinatol. 34, 130-135 (2014).

10. Mascoll-Robertson, K. K., Viscardi, R. M. \& Woo, H. C. The objective use of pulse oximetry to predict respiratory support transition in preterm infants: an observational pilot study. Respir. Care 61, 416-422 (2016).

11. Dani, C. Automated control of inspired oxygen ( $\mathrm{FiO} 2)$ in preterm infants: literature review. Pediatr. Pulmonol. 54, 358-363 (2019). 